WUE-ITP-2001-004

hep-ph/0101266

January 2001

\title{
Light Charged Higgs Boson and Supersymmetry
}

\author{
C. Panagiotakopoulos ${ }^{a}$ and A. Pilaftsis ${ }^{b}$ \\ a Physics Division, School of Technology, Aristotle University of Thessaloniki, \\ 54006 Thessaloniki, Greece \\ ${ }^{b}$ Institut für Theoretische Physik und Astrophysik, Universität Würzburg, \\ Am Hubland, 97074 Würzburg, Germany
}

\begin{abstract}
A possible discovery of a relatively light charged Higgs boson $H^{+}$in near future experiments, with a mass $M_{H^{+}} \lesssim 110 \mathrm{GeV}$, together with the present LEP2 direct limits on the chargino and neutral Higgs sectors, would disfavour the minimal supersymmetric standard model as well as its frequently discussed next-to-minimal supersymmetric extension. We show that a supersymmetric origin can naturally be ascribed to the existence of such a light charged Higgs scalar within the context of the recently introduced minimal nonminimal supersymmetric standard model.
\end{abstract}


Supersymmetry (SUSY) appears to be a compelling ingredient of string theories which are expected to successfully describe the Planck-scale dynamics, thereby aspiring to unify all fundamental forces in nature, including gravity. For these reasons, low-energy realizations of SUSY softly broken at $0.1-1 \mathrm{TeV}$ energies, such as the Minimal Supersymmetric Standard Model (MSSM) and its minimal extensions, are considered to be the best-motivated models [1] of physics beyond the Standard Model (SM). Most interestingly, such low-energy realizations of SUSY exhibit gauge-coupling unification [2] and can solve, at least technically, the problem of perturbative stability of radiative effects between the soft SUSY-breaking scale $M_{\mathrm{SUSY}} \sim 1 \mathrm{TeV}$ and the Planck mass $M_{\mathrm{P}}$. These appealing properties of low-energy SUSY might be considered to mainly emanate from the doubling of the particle spectrum of the SM; the theory introduces a new fermion (boson) for each SM boson (fermion), its so-called superpartner. Superpartners have typical masses of the order of the soft SUSY-breaking scale $M_{\text {SUSY }}$ and should be heavier than $\sim 100 \mathrm{GeV}$, for phenomenological reasons [3]. In addition, within the framework of SUSY, the holomorphicity of the superpotential together with the requirement of cancellation of the triangle gauge anomalies entail that the SM Higgs sector itself must be augmented by at least one Higgs doublet of opposite hypercharge. To be specific, low-energy SUSY models include a minimal set of two Higgs iso-doublets and so necessarily predict the existence of at least one (doublet) charged Higgs boson, $H^{ \pm}$, in addition to a number of neutral Higgs particles. As we will see in this note, the mass of $\mathrm{H}^{+}$introduces a new scale into the neutral Higgs-boson mass spectrum and can play a key rôle in distinguishing among different minimal models of electroweak-scale SUSY.

In the decoupling limit of a heavy charged Higgs boson, low-energy SUSY models make a definite prediction for the mass of that neutral Higgs boson $H$, which is predominantly responsible for the spontaneous breaking of the SM gauge group. In other words, for large values of $M_{H^{+}}$, e.g. $M_{H^{+}} \sim 1 \mathrm{TeV}$, the mass of the SM-like $H$ boson reaches a calculable model-dependent maximum [4]. For instance, in the MSSM, with radiative effects included [5], recent computations [6, [7,8] lead to the upper limit: $M_{H} \lesssim 110$ (130) GeV for $\tan \beta \approx 2(\gtrsim 10)$, where $\tan \beta$ is the ratio of the vacuum expectation values (VEV's) of the two Higgs doublets. On the other hand, in the frequently discussed extension of the MSSM known as the Next-to-Minimal Supersymmetric Standard Model (NMSSM) [9], the maximum of the corresponding $H$-boson mass may increase by an amount of $\sim 30 \mathrm{GeV}$, for $\tan \beta \approx 2$, while it remains unaffected for large values of $\tan \beta$ [10. Apart from $\tan \beta$, however, the Higgs-boson mass spectrum depends very sensitively on the actual value of $M_{H^{+}}$and the stop-mixing parameter $X_{t}=A_{t}-\mu / \tan \beta$, where $A_{t}$ is the soft SUSYbreaking Yukawa coupling to stops and $\mu$ the mixing parameter of the two Higgs-doublet superfields $\widehat{H}_{1}$ and $\widehat{H}_{2}$. For example, for $M_{H^{+}} \lesssim 110 \mathrm{GeV}$, the masses of the lightest CPeven Higgs boson $h$ and the CP-odd scalar $A$ are predicted to be both less than $\sim 80 \mathrm{GeV}$, almost independently of $\tan \beta$, provided no unusually large values of $|\mu|$ are considered, e.g. for $|\mu| \lesssim 2 M_{\mathrm{SUSY}}$. However, such a scenario is disfavoured by the latest LEP2 data [11], 
since it predicts an enhanced $Z h A$-coupling, whenever the $h Z Z$-coupling is suppressed, and hence would have been detected in the corresponding $Z h A$ channel. As has been explicitly demonstrated in [12], a similar negative conclusion may be reached in the NMSSM as well, for $M_{H^{+}} \lesssim 110 \mathrm{GeV}$. In this model, the SM-like Higgs boson $H$ always comes out to be lighter than $H^{+}$, provided the effectively generated $\mu$-parameter lies in the phenomenologically favoured range, $|\mu| \gtrsim 100 \mathrm{GeV}$, as is suggested by the non-observation of chargino production at LEP2 [3, 13]. . .

Given the difficulty that the MSSM and NMSSM cannot easily accommodate a charged Higgs boson $H^{+}$lighter than the SM-like neutral Higgs boson $H$, one may now raise the following question: Should such a light charged particle, with $M_{H^{+}} \lesssim 110 \mathrm{GeV}$, be observed, e.g. at the upgraded Tevatron collider, is it then possible to ascribe to it a supersymmetric origin within a minimal SUSY extension of the SM? In this note we address this important question in the affirmative within the framework of the recently introduced Minimal Nonminimal Supersymmetric Standard Model (MNSSM) [12,16].

In the MNSSM the $\mu$-parameter is promoted to a chiral singlet superfield $\widehat{S}$, and all linear, quadratic and cubic operators involving only $\widehat{S}$ are absent from the renormalizable superpotential; $\widehat{S}$ enters through the single term $\lambda \widehat{S} \widehat{H}_{1} \widehat{H}_{2}$. The crucial difference between the MNSSM and the NMSSM lies in the fact that the cubic term $\frac{1}{3} \kappa \widehat{S}^{3}$ does not appear in the renormalizable superpotential of the former. This particularly simple form of the renormalizable MNSSM superpotential may be enforced by discrete $R$-symmetries, such as $\mathcal{Z}_{5}^{R}$ 16, 12 and $\mathcal{Z}_{7}^{R}$ [12. These discrete $R$-symmetries, however, must be extended to the gravity-induced non-renormalizable superpotential and Kähler potential terms as well. Here, we consider the scenario of $N=1$ supergravity spontaneously broken by a set of hidden-sector fields at an intermediate scale. Within this framework of SUSY-breaking, we have been able to show [12] that the above $R$-symmetries are sufficient to guarantee the appearance of the potentially dangerous tadpole $t_{S} S$, with $t_{S} \sim\left(1 / 16 \pi^{2}\right)^{n} M_{\mathrm{P}} M_{\mathrm{SUSY}}^{2}$, at loop levels $n$ higher than 5. As a consequence, we have $\left|t_{S}\right| \lesssim 1-10 \mathrm{TeV}^{3}$, and therefore the gauge hierarchy does not get destabilized. Notice that the so-generated tadpole $t_{S} S$ together with the soft SUSY-breaking mass term $m_{S}^{2} S^{*} S$ lead to a VEV for $S,\langle S\rangle=\frac{1}{\sqrt{2}} v_{S}$, of order $M_{\text {SUSY }}$. The latter gives rise to a $\mu$-parameter at the required electroweak scale, i.e. $\mu=-\frac{1}{\sqrt{2}} \lambda v_{S} \sim M_{\mathrm{SUSY}}$, thus offering a natural explanation for the origin of the $\mu^{-}$ parameter. Finally, since the effective tadpole term $t_{S} S$ explicitly breaks the continuous Peccei-Quinn symmetry governing the remaining renormalizable Lagrangian of the MNSSM, the theory naturally avoids the presence of a phenomenologically excluded weakscale axion.

${ }^{*}$ Throughout the paper, we shall not consider possible indirect constraints on the $H^{+}$-boson mass from $b \rightarrow s \gamma$ and other observables involving $B$ mesons, as the derived limits sensitively depend on several other model-dependent parameters of the theory, such as the sign of $\mu$ [14] and the low-energy flavour-mixing structure of the squark sector [15]. 
The MNSSM predicts, in addition to the charged Higgs scalar $H^{+}$, five neutral Higgs bosons. Under the assumption of $\mathrm{CP}$ invariance, three of the neutral Higgs particles, denoted as $H_{1}, H_{2}$ and $H_{3}$ in order of increasing mass, are CP-even, while the other two, $A_{1}$ and $A_{2}$ (with $M_{A_{1}}<M_{A_{2}}$ ), are CP-odd. Nevertheless, since the tadpole $\left|t_{S}\right|$ naturally takes values of the order of $1-10 \mathrm{TeV}^{3}$, the Higgs-boson mass spectrum of the MNSSM simplifies considerably: the heaviest states $H_{3}$ and $A_{2}$, with $M_{H_{3}}^{2} \approx M_{A_{2}}^{2} \approx \lambda t_{S} / \mu$, decouple as singlets from the remaining Higgs sector. Then, the masses of $H^{+}$and $A_{1}$ satisfy the relation

$$
M_{A_{1}}^{2} \approx M_{a}^{2}=M_{H^{+}}^{2}-M_{W}^{2}+\frac{1}{2} \lambda^{2} v^{2}-\delta_{\text {rem }},
$$

where $M_{W}=g_{w} v / 2$ is the $W$-boson mass and $\delta_{\text {rem }}$ contains the radiative corrections which may be approximately determined by [17, 12, 18]

$$
\delta_{\text {rem }} \approx-\frac{3 h_{t}^{4}}{32 \pi^{2}} \frac{\mu^{2} v^{2}}{m_{\tilde{t}_{1}}^{2}+m_{\tilde{t}_{2}}^{2}},
$$

where $\tilde{t}_{1}$ and $\tilde{t}_{2}$ are the stop mass eigenstates. Notice that the relation (11) is very analogous to the one known from the MSSM. Specifically, the squared mass term $M_{a}^{2}$ enters the nondecoupled $2 \times 2$ CP-even mass matrix the same way as the squared mass of the would-be CP-odd Higgs scalar in the MSSM. As opposed to the MSSM, however, the presence of the term $\frac{1}{2} \lambda^{2} v^{2}$ in Eq. (四) implies that the $H^{+}$boson can become even lighter than $A_{1}$, for $\lambda \sim g_{w} ; H^{+}$can be as light as its experimental lower bound, $M_{H^{+}} \sim 80 \mathrm{GeV}$ [3. [1]. As an important consequence, the $H^{+}$boson can naturally be lighter than the SM-like Higgs boson $H$. As we will see, this prediction is very unique for the MNSSM. In the MSSM, such a result may be achieved for unconventionally large values of $|\mu|$, in which case $\delta_{\text {rem }}$ in Eq. (2) will start playing a very analogous rôle as the term $\frac{1}{2} \lambda^{2} v^{2}$ in Eq. (11) does for the MNSSM.

For our phenomenological discussion, we denote with $g_{H_{i} Z Z}, g_{H_{i} W W}$ and $g_{H_{i} A_{j} Z}$ the strength of the effective $H_{i} W W-, H_{i} Z Z$ - and $H_{i} A_{j} Z$ - couplings, respectively normalized to the SM values of the $H W W$-, $H Z Z$ - and $H Z G^{0}$ - couplings, where $G^{0}$ is the would-be Goldstone boson of $Z$. These SM-normalized effective couplings obey the unitarity relations: $\sum_{i=1}^{3} g_{H_{i} V V}^{2}=1$ and $\sum_{i=1}^{3} \sum_{j=1}^{2} g_{H_{i} A_{j} Z}^{2}=1$, with $V=W, Z$. Moreover, as a consequence of a large $\left|t_{S}\right|$, the effective Higgs-to-gauge-boson couplings satisfy the approximate equalities

$$
g_{H_{1} V V}^{2} \approx g_{H_{2} A_{1} Z}^{2}, \quad g_{H_{2} V V}^{2} \approx g_{H_{1} A_{1} Z}^{2}
$$

which are essentially identical to the corresponding complementarity equalities of the MSSM. We should remark that the relations (33) are not valid in the NMSSM, since the states $H_{3}$ and $A_{2}$ do not decouple as singlets from the lightest Higgs sector in the latter model.

Our study of the MNSSM Higgs-boson mass spectrum in the decoupling limit of a large $\left|t_{S}\right|$ utilizes renormalization-group (RG) techniques developed in [6,8,19] for the 
MSSM case and so improves in several respects earlier considerations in the NMSSM, in which an analogous decoupling limit is lacking. Specifically, in addition to the oneloop stop $(\tilde{t})$ and sbottom $(\tilde{b})$ corrections, our RG improvement consists in including twoloop leading logarithms induced by QCD and top- $(t)$ and bottom- $(b)$ quark Yukawa interactions. Further, we take into account the leading logarithms originating from gaugino and higgsino one-loop graphs [17], as well as we implement the potentially large two-loop contributions induced by the one-loop $\tilde{t}$ - and $\tilde{b}$ - squark thresholds in the $t$ - and $b$ - quark Yukawa couplings [8].

In the MNSSM and NMSSM, the SM-normalized effective couplings $g_{H_{i} V V}$ and the CP-even Higgs-boson masses $M_{H_{i}}$ satisfy an important sum rule:

$$
\begin{aligned}
\sum_{i=1}^{3} g_{H_{i} V V}^{2} & M_{H_{i}}^{2}=\left(M_{Z}^{2} \cos ^{2} 2 \beta+\frac{1}{2} \lambda^{2} v^{2} \sin ^{2} 2 \beta\right)\left(1-\frac{3 h_{t}^{2}}{8 \pi^{2}} t\right) \\
+ & \frac{3 h_{t}^{4} v^{2} \sin ^{4} \beta}{8 \pi^{2}}\left\{\left(1+\frac{4 \alpha_{s}}{3 \pi} \frac{X_{t}}{M_{\mathrm{SUSY}}}\right)\left[t+\frac{X_{t}^{2}}{M_{\mathrm{SUSY}}^{2}}\left(1-\frac{X_{t}^{2}}{12 M_{\mathrm{SUSY}}^{2}}\right)\right]\right. \\
+ & \left.\frac{1}{16 \pi^{2}}\left(\frac{3}{2} h_{t}^{2}-32 \pi \alpha_{s}\right)\left[\frac{2 X_{t}^{2}}{M_{\mathrm{SUSY}}^{2}}\left(1-\frac{X_{t}^{2}}{12 M_{\mathrm{SUSY}}^{2}}\right) t+t^{2}\right]\right\}+\mathcal{O}\left(\frac{X_{t}^{6}}{M_{\mathrm{SUSY}}^{6}}\right),
\end{aligned}
$$

where $t=\ln \left(M_{\mathrm{SUSY}}^{2} / m_{t}^{2}\right)$, and the strong fine structure constant $\alpha_{s}$, the $t$-quark Yukawa coupling $h_{t}$ and the SM VEV $v$ are to be evaluated at $m_{t}$. The mass-coupling sum rule (4) is independent of $M_{H^{+}}$and makes the definite prediction that the mass of the neutral Higgs boson $H$ with SM coupling to the $Z$ boson, $g_{H Z Z}^{2} \approx 1$, is completely specified by a model-dependent value determined from the RHS of Eq. (4). It can thus be estimated from Eq. (州) that in the MNSSM and NMSSM, the SM-like Higgs-boson mass can reach a maximum of $\sim 142 \mathrm{GeV}$, for $\tan \beta=2, \lambda=0.65, M_{\mathrm{SUSY}} \approx 1 \mathrm{TeV}$ and $X_{t} \approx 2.45 \mathrm{TeV}$. In addition, one should observe that the mass-coupling sum rule holds true for the MSSM case as well, after setting $\lambda=0$. The analytic result of the RHS of Eq. (四) is then in agreement with the one computed in [8], after one follows the suggested RG approach to implementing stop threshold effects on the $t$-quark Yukawa coupling.

It is now very interesting to quote results of variants of the MNSSM that could be probed at LEP2 and especially at the upgraded Tevatron collider in the immediate future. For definiteness, in our numerical estimates, we fix the soft SUSY-breaking squark masses to $M_{\mathrm{SUSY}}=1 \mathrm{TeV}$, and the $\mathrm{U}(1)_{Y}, \mathrm{SU}(2)_{L}$ and $\mathrm{SU}(3)_{c}$ gaugino masses to $m_{\widetilde{B}}=m_{\widetilde{W}}=$ $0.3 \mathrm{TeV}$ and $m_{\tilde{g}}=1 \mathrm{TeV}$, respectively. Motivated by the recently observed excess of events for a SM-like Higgs boson of a mass $\sim 115 \mathrm{GeV}$ at LEP2 [20], we choose in Tables 1$]$ and 2 the mass of the second lightest CP-even Higgs boson $H_{2}$ to be $M_{H_{2}} \approx 115 \mathrm{GeV}$, with $g_{H_{2} Z Z}^{2} \approx 0.9$. For the lightest Higgs boson $H_{1}$, whose squared effective coupling to the $Z$ boson is necessarily $g_{H_{1} Z Z}^{2} \approx 1-g_{H_{2} Z Z}^{2} \approx 0.1$, we assume a lower mass, i.e. $M_{H_{1}} \approx 95 \mathrm{GeV}$, compatible with the present LEP2 data 11. In Table 11 we consider the zero stop-mixing scenario, i.e. $X_{t}=0$, and choose $\lambda t_{S} / \mu=5 \mathrm{TeV}^{2}$. We find that the 


\begin{tabular}{lcccc}
\hline \hline$M_{H^{+}}[\mathrm{GeV}]$ & $\tan \beta$ & $\lambda$ & $\mu[\mathrm{GeV}]$ & $M_{A_{1}}[\mathrm{GeV}]$ \\
\hline 70 & 1.74 & 0.614 & -574 & 101.8 \\
80 & 2.82 & 0.542 & -331 & 95.7 \\
90 & 4.93 & 0.488 & -224 & 95.4 \\
100 & 7.70 & 0.424 & -229 & 95.9 \\
110 & 10.86 & 0.338 & -290 & 96.2 \\
120 & 14.34 & 0.205 & -410 & 96.4 \\
125.2 & 16.35 & 0. & -825 & 96.5 \\
\hline \hline
\end{tabular}

Table 1: Predictions of the MNSSM [21], using as inputs: $X_{t}=0, A_{t}=A_{b}, M_{\mathrm{SUSY}}=1 \mathrm{TeV}$, $m_{\widetilde{B}}=m_{\widetilde{W}}=0.3 \mathrm{TeV}, m_{\tilde{g}}=1 \mathrm{TeV}, \lambda t_{S} / \mu=5 \mathrm{TeV}^{2}, M_{H_{1}} \approx 95 \mathrm{GeV}, M_{H_{2}} \approx 115 \mathrm{GeV}$, $g_{H_{1} Z Z}^{2} \approx 0.1$ and $g_{H_{2} Z Z}^{2} \approx 0.9$.

mass of the charged Higgs boson may naturally lie below $110 \mathrm{GeV}$, for reasonable values of the MNSSM parameters. In particular, we obtain $\lambda \lesssim 0.65$, for $\tan \beta \gtrsim 2$. Interestingly enough, such a range of $\lambda$ values is also consistent with the requirement of perturbativity of the MNSSM up to the gauge-coupling unification scale $M_{\mathrm{U}} \sim 10^{16} \mathrm{GeV}$ [2,10]. Also, in accordance with our earlier discussion, we observe that the $H^{+}$boson must be as heavy as $125 \mathrm{GeV}$ in the MSSM limit $\lambda \rightarrow 0$, i.e. heavier than the SM-like Higgs boson $H_{2}$. In Table 2, we select $\lambda t_{S} / \mu=1.5 \mathrm{TeV}^{2}$ and use the value of maximal stop mixing, $X_{t} \approx \sqrt{6} M_{\mathrm{SuSY}}$, characterized by the fact that the radiative effects given in Eq. (雨) get approximately maximized. We arrive at the very same conclusion, namely the $\mathrm{H}^{+}$boson can be lighter than $\sim 110 \mathrm{GeV}$ and so lighter than the SM-like Higgs boson $H_{2}$. This particular feature of the MNSSM is also reflected in Fig. 1, where we show numerical values of $H_{1^{-}}$and $H_{2^{-}}$ boson masses and of their squared effective couplings to the $Z$ boson as functions of $\mu$, for three variants of the MNSSM from Table 1: $M_{H^{+}}=80,100$ and $120 \mathrm{GeV}$. We observe that the aforementioned LEP2-motivated scenario of a SM-like Higgs boson may be accounted for by a wide range of $\mu$ values.

An interesting alternative emerges if one of the two non-decoupled CP-even Higgs bosons, e.g. $H_{2}$, has a mass $M_{H_{2}} \approx 115 \mathrm{GeV}$ with $g_{H_{2} Z Z}^{2} \approx 1$, while the other one, $H_{1}$, does not couple to the $Z$ boson but has $g_{A_{1} H_{1} Z}^{2} \approx 1$, and vice versa. Such an alternative is easily compatible with the LEP2 data, as long as the mass inequality constraint, $M_{H_{1}}+M_{A_{1}} \gtrsim$ $170 \mathrm{GeV}$ [11, is met. Assuming $\lambda t_{S} / \mu=2 \mathrm{TeV}^{2}$, the above scenario may be realized for a wide range of charged Higgs-boson masses between 60 and $110 \mathrm{GeV}$, and for both zero and maximal stop mixing. For instance, for $X_{t}=0$, such a kinematic dependence insensitive to $M_{H^{+}}$may be obtained for $\tan \beta=2.5, \lambda=0.623$ and $\mu \approx-283 \mathrm{GeV}$, while for $X_{t}=2.45 \mathrm{TeV}$, one may choose $\tan \beta=5, \lambda=0.645$ and $\mu \approx-393 \mathrm{GeV}$. In Fig. 2, we display the dependence of the $H_{1^{-}}$and $H_{2^{-}}$boson masses and of their squared 


\begin{tabular}{lcccc}
\hline \hline$M_{H^{+}}[\mathrm{GeV}]$ & $\tan \beta$ & $\lambda$ & $\mu[\mathrm{GeV}]$ & $M_{A_{1}}[\mathrm{GeV}]$ \\
\hline 60 & 2.24 & 0.649 & -441 & 102.8 \\
70 & 3.28 & 0.590 & -402 & 97.8 \\
80 & 5.15 & 0.541 & -397 & 96.7 \\
90 & 7.73 & 0.489 & -424 & 96.7 \\
100 & 10.7 & 0.427 & -481 & 97.1 \\
110 & 14.0 & 0.348 & -585 & 97.7 \\
120 & 17.65 & 0.249 & -810 & 99.0 \\
\hline \hline
\end{tabular}

Table 2: Predictions of the MNSSM [21], using the same inputs as in Table 1, with the exception that $X_{t}=2.45 \mathrm{TeV}$ and $\lambda t_{S} / \mu=1.5 \mathrm{TeV}^{2}$.

effective couplings to the $Z$ boson as a function of the $\mu$-parameter, for $M_{H^{+}}=80,100$ and $120 \mathrm{GeV}$ in the aforementioned variant of the MNSSM with $X_{t}=0$. We observe again that the $H^{+}$boson can be lighter than $\sim 110 \mathrm{GeV}$ and therefore lighter than the SM-like Higgs boson. In addition, we notice that there exists a SM-like Higgs boson for a very wide range of $\mu$ values and, only for a very short interval of $\mu$, the $H_{1}$ and $H_{2}$ bosons interchange their couplings to the $Z$ boson, while they are degenerate in mass. Finally, we should reiterate the fact that analogous possibilities are present in the MSSM for large values of $|\mu|$. In agreement with our earlier observation, we find that the $H^{+}$boson can be as light as $100 \mathrm{GeV}$, with $M_{H_{2}} \approx 115 \mathrm{GeV}, M_{H_{1}} \approx 82.3 \mathrm{GeV}, M_{A_{1}} \approx 92.6 \mathrm{GeV}$ and $g_{H_{2} Z Z}^{2} \approx g_{H_{1} A_{1} Z}^{2} \approx 1$, if the large value of $|\mu|, \mu \approx-3.97 \mathrm{TeV}$, together with $\tan \beta=12.3$ and $X_{t}=1 \mathrm{TeV}$, is employed.

In the NMSSM the situation is different. The charged Higgs-boson mass $M_{H^{+}}$exhibits a strong monotonic dependence on the $\mu$-parameter; $|\mu|$ gets rapidly smaller for smaller values of $M_{H^{+}}$. This generic feature of the NMSSM may mainly be attributed to the fact [12] that no analogous decoupling limit due to a large $\left|t_{S}\right|$ exists in this model. In particular, unlike the MNSSM case, no actual use of the presence of the contribution $\frac{1}{2} \lambda^{2} v^{2}$ in the Higgs-boson mass matrices can be made in the NMSSM. In fact, we find that it is always $M_{H^{+}} \gtrsim 110 \mathrm{GeV}$, for phenomenologically favoured values of $\mu$, i.e. for $|\mu| \gtrsim 100 \mathrm{GeV}$ [3, 13], it] assuming that the theory stays perturbative up to $M_{\mathrm{U}}$. If the $H^{+}$boson becomes heavier than the neutral SM-like Higgs boson $H$, the phenomenological distinction between the NMSSM and MNSSM is getting more difficult and additional experimental information would be necessary, such as the testing of the complementarity coupling relations of Eq. (3).

To summarize: the renormalizable low-energy sector of the MNSSM in the decoupling limit due to a large $\left|t_{S}\right|$ has effectively one parameter more than the corresponding one of

${ }^{\dagger}$ If this last constraint on the $\mu$-parameter is lifted, then charged Higgs-boson masses as low as $90 \mathrm{GeV}$ might be possible in the NMSSM [22]. 
the (CP-conserving) MSSM, namely the coupling $\lambda$. In fact, in the MNSSM the natural size of the higher-loop generated tadpole parameter $\left|t_{S}\right|$ is of order $1-10 \mathrm{TeV}^{3}$. For unsuppressed values of $\lambda, t_{S}$ leads to masses of the order of $1 \mathrm{TeV}$ for the heaviest CP-even and CPodd Higgs scalars $H_{3}$ and $A_{2}$, so these states decouple as heavy singlets giving rise to an active low-energy Higgs sector consisting only of doublet-Higgs fields, closely analogous to the one of the MSSM. The latter should be contrasted with the NMSSM case, where no analogous decoupling limit due to a large $\left|t_{S}\right|$ exists in this model. Most strikingly, the MNSSM may also predict a light charged Higgs boson, which can be even lighter than the SM-like Higgs boson $H$. We should stress again that in the light of the present LEP2 data, such a prediction cannot be naturally obtained in the MSSM or NMSSM. In the same vein, we note that it would be very interesting to study as well as identify the compelling lowenergy structure of other SUSY extensions of the SM that could lead to the inverse mass hierarchy $M_{H^{+}} \ll M_{H}$. From our discussion, however, it is obvious that the MNSSM trully represents the simplest and most economic non-minimal supersymmetric model proposed in the literature after the MSSM. In conclusion, it is very important that the upgraded Tevatron collider has the physics potential to probe the exciting hypothesis of a light charged Higgs boson in top decays $t \rightarrow H^{+} b$ [23] and analyze its possible consequences within the framework of the MNSSM.

\section{Acknowledgements}

The work of A.P. is supported in part by the Bundesministerium für Bildung und Forschung under the contract number 05HT9WWA9. 


\section{References}

[1] For reviews, see, H.-P. Nilles, Phys. Rep. 110 (1984) 1; H.E. Haber and G.L. Kane, Phys. Rep. 117 (1985) 75; A.B. Lahanas and D.V. Nanopoulos, Phys. Rep. 145 (1987) 1; J.F. Gunion, H.E. Haber, G.L. Kane and S. Dawson, "The Higgs Hunter's Guide," (Addison-Wesley, Reading, MA, 1990).

[2] See, e.g., M. Carena, S. Pokorski and C.E.M. Wagner, Nucl. Phys. B406 (1993) 59.

[3] Review of Particle Physics (D.E. Groom et al.), Eur. Phys. J. C15 (2000) 1.

[4] For example, see, J.R. Espinosa and M. Quirós, Phys. Rev. Lett. 81 (1998) 516.

[5] J. Ellis, G. Ridolfi and F. Zwirner, Phys. Lett. B257 (1991) 83; M.S. Berger, Phys. Rev. D41 (1990) 225; Y. Okada, M. Yamaguchi and T. Yanagida, Prog. Theor. Phys. 85 (1991) 1; H.E. Haber and R. Hempfling, Phys. Rev. Lett. 66 (1991) 1815.

[6] M. Carena, M. Quirós and C.E.M. Wagner, Nucl. Phys. B461 (1996) 407; M. Carena, S. Mrenna and C.E.M. Wagner, Phys. Rev. D60 (1999) 075010.

[7] S. Heinemeyer, W. Hollik and G. Weiglein, Phys. Rev. D58 (1998) 091701.

[8] M. Carena, H.E. Haber, S. Heinemeyer, W. Hollik, C.E.M. Wagner and G. Weiglein, Nucl. Phys. B580 (2000) 29; J.R. Espinosa and R.-J. Zhang, JHEP 0003 (2000) 026.

[9] P. Fayet, Nucl. Phys. B90 (1975) 104; H.-P. Nilles, M. Srednicki and D. Wyler, Phys. Lett. B120 (1983) 346; A.B. Lahanas, Phys. Lett. B124 (1983) 341. For reviews, see, M. Drees, Int. J. Mod. Phys. A4 (1989) 3635; F. Franke and H. Fraas, Int. J. Mod. Phys. A12 (1997) 479.

[10] T. Elliott, S.F. King and P.L. White, Phys. Rev. D49 (1994) 2435. For a recent computation, see, U. Ellwanger and C. Hugonie, hep-ph/9909260.

[11] ALEPH, DELPHI, L3 and OPAL Collaborations (P. Bock et al.), CERN-EP-2000-055; OPAL Collaboration (G. Abbiendi et al.), CERN-EP-2000-092, hep-ex/0007040.

[12] C. Panagiotakopoulos and A. Pilaftsis, Phys. Rev. D63 (2001) 055003.

[13] N. Ghodbane, S. Katsanevas, I. Laktineh and J. Rosiek, hep-ph/0012031.

[14] S. Bertolini, F. Borzumati, A. Masiero and G. Ridolfi, Nucl. Phys. B353 (1991) 591; R. Barbieri and G.F. Giudice, Phys. Lett. B309 (1993) 86; R. Garisto and J.N. Ng, Phys. Lett. B315 (1993) 372.

[15] Y. Nir and N. Seiberg, Phys. Lett. B309 (1993) 337; D. Choudhury, F. Eberlein, A. König, J. Louis and S. Pokorski, Phys. Lett. B342 (1995) 180. 
[16] C. Panagiotakopoulos and K. Tamvakis, Phys. Lett. B469 (1999) 145.

[17] H. Haber and R. Hempfling, Phys. Rev. D48 (1993) 4280.

[18] For a RG-improved computation of $M_{H^{+}}$in the MSSM, see, A. Pilaftsis and C.E.M. Wagner, Nucl. Phys. B553 (1999) 3; M. Carena, J. Ellis, A. Pilaftsis and C.E.M. Wagner, Nucl. Phys. B586 (2000) 92; Phys. Lett. B495 (2000) 155.

[19] J. Kodaira, Y. Yasui and K. Sasaki, Phys. Rev. D50 (1994) 7035; J.A. Casas, J.R. Espinosa, M. Quirós and A. Riotto, Nucl. Phys. B436 (1995) 3; Nucl. Phys. B439 (1995) 466 (Erratum).

[20] ALEPH Collaboration (R. Barate et al.), Phys. Lett. B495 (2000) 1; L3 Collaboration (M. Acciarri et al.), Phys. Lett. B495 (2000) 18.

[21] Numerical values are obtained by the Fortran code mnssm which is available from http://pilaftsi.home.cern.ch/pilaftsi/.

[22] M. Drees, E. Ma, P.N. Pandita, D.P. Roy and S.K. Vempati, Phys. Lett. B433 (1998) 346.

[23] T. Affolder et al., The CDF Collaboration, http://www-cdf.fnal.gov/physics/ preprints/cdf5124_charged_higgs_prd.ps. 


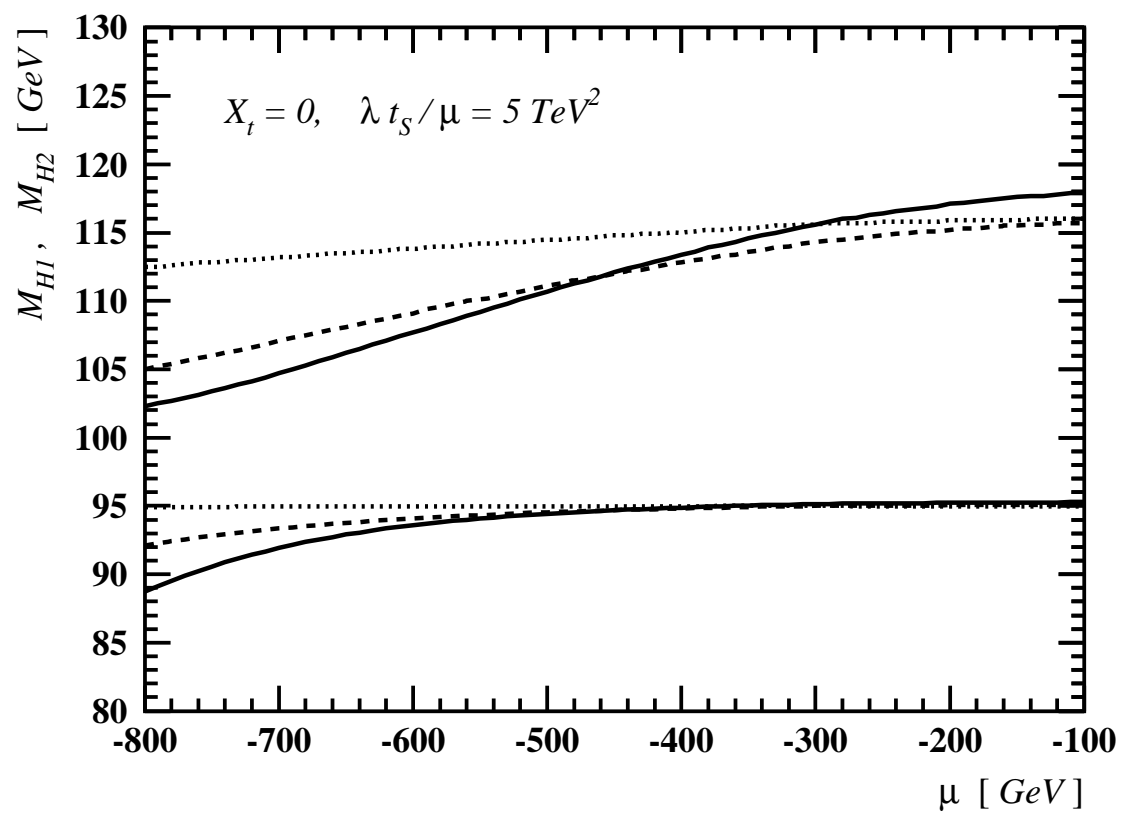

(a)

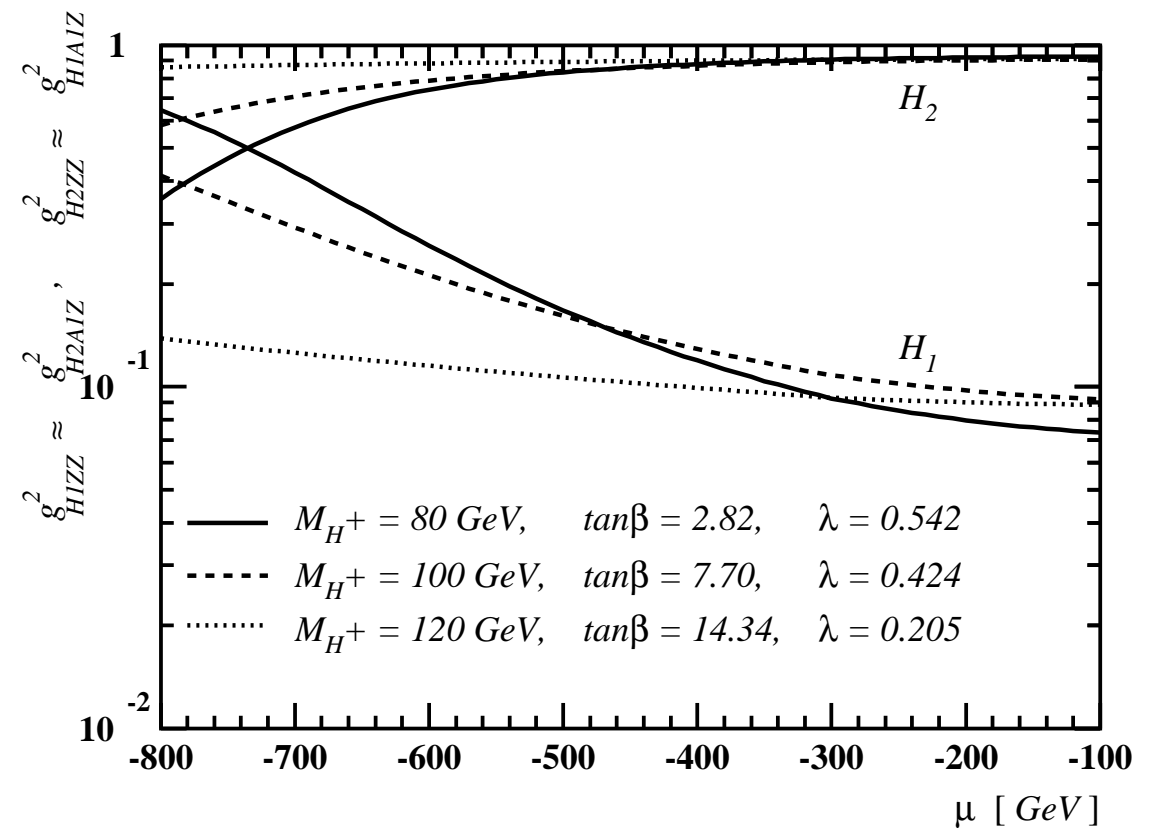

(b)

Figure 1: Numerical predictions for (a) $M_{H_{1}}$ and $M_{H_{2}}$, and (b) $g_{H_{1} Z Z}^{2}$ and $g_{H_{2} Z Z}^{2}$, as functions of $\mu$ in the MNSSM. 


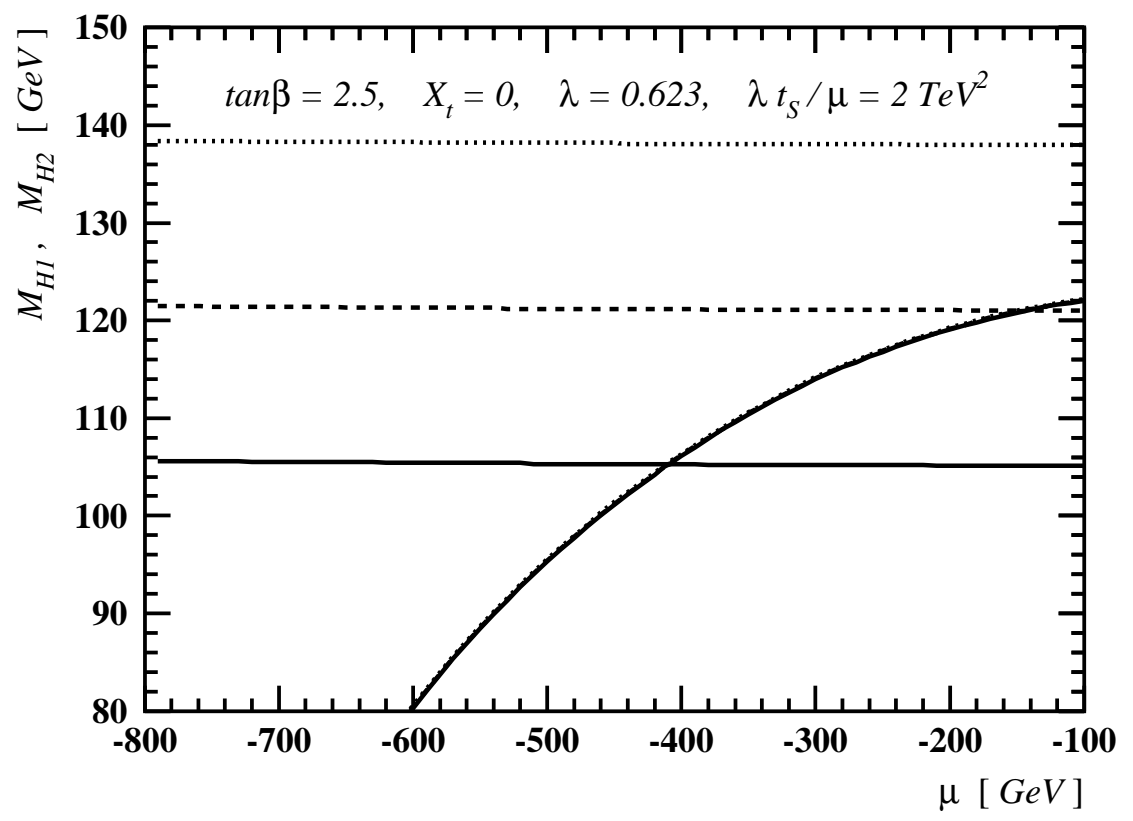

(a)

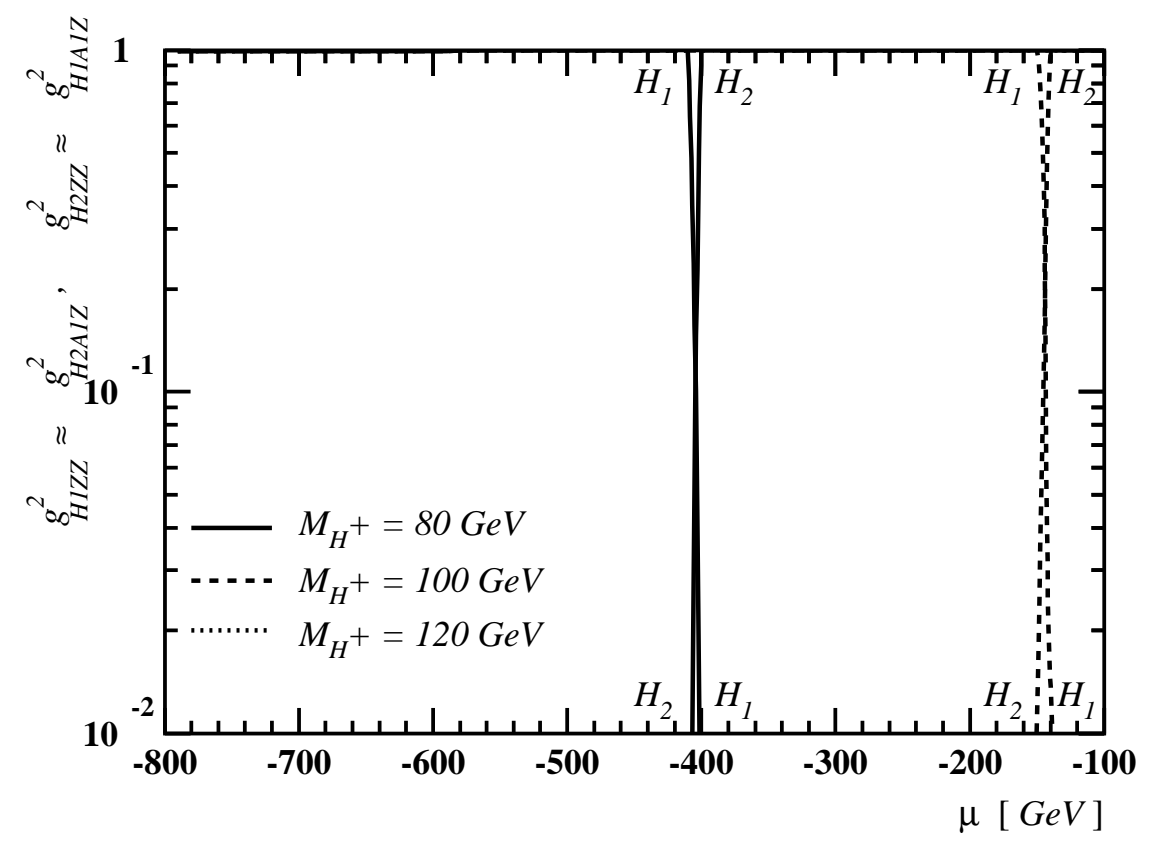

(b)

Figure 2: Numerical values of (a) $M_{H_{1}}$ and $M_{H_{2}}$, and (b) $g_{H_{1} Z Z}^{2}$ and $g_{H_{2} Z Z}^{2}$, as functions of $\mu$ in the MNSSM. 